\title{
EDITORIAL
}

\section{Special Topic: Biomedical Application of DNA-Assembled Nanostructure}

\author{
Jun-Jie Zhu ${ }^{1}$ \\ Published online: 29 May 2021 \\ (c) The Nonferrous Metals Society of China 2021
}

Pathological features of diseases are typically accompanied with certain upregulated biomarkers, such as ions, nucleic acids, small molecules and proteins. Highly sensitive detecting tools with acceptable compatibility are of paramount importance for the precise diagnose of diseases. However, the sensitivity, accuracy and biosafety of probes still remain a challenge, which persuade researchers to explore more applicable materials and platforms to address these issues.

The past few decades have witnessed the burgeoning evolution of DNA nanotechnology in the field of biosensing, bioimaging and biomedicine owing to its programmability, predictability and biocompatibility. As a kind of versatile building block, nucleic acid could be assembled into diverse structures with predictable configurations, including DNA origami, DNA hydrogel and multifunctional DNA nanostructure that can provide controllable carriers to deliver diagnostic or therapeutic reagents. Functional DNA-like aptamer has high affinity to certain molecules or cellular receptors, which can be utilized to increase the detection accuracy of analysts. Meanwhile, DNA signal amplification based on polymerase chain reaction (PCR), rolling circle amplification (RCA) or hybridization chain reaction (HCR) is capable of analyzing targeted substances in low abundance with ameliorated sensitivity and selectivity. More importantly, as a naturally derived material, DNA is endowed with good compatibility that holds promise for clinical translation.

Concerning the current development and potential application of DNA nanotechnology, Journal of Analysis and Testing organized this special issue including five review papers and four research articles to provide readers with a comprehensive understanding in this field. Prof. Chao and coworkers from Nanjing University of Posts and Telecommunications reviewed the recent progress of nucleic

Jun-Jie Zhu

jjzhu@nju.edu.cn

1 State Key Laboratory of Analytical Chemistry for Life Science, School of Chemistry and Chemical Engineering, Nanjing University, Nanjing 210023, China acids-based functional nanomaterials in the application of bioimaging. Taking advantage of different amplification mechanism and stimuli-responsiveness, nucleic acids-based nanomaterial could achieve the time-spatial controlled imaging of intracellular targets. Prof. Bi and colleagues from Qingdao University provided an impressive review of DNA nanotechnology for multimodal synergistic theranostics, highlighting the superadditive effect of DNA-aided bioimaging and diseases therapy. Focusing on a specific type of DNA structure, Prof. Zuo and Prof. Wang from Southwest University summarized the recent application of DNA hydrogel in biosensing, biomedicine and cancer therapy and indicated the future direction in these fields. Besides, Prof. Nie from Hunan University introduced the advances in the integration of nucleic acid nanotechnology into clustered regularly interspaced short palindromic repeats (CRISPR) and CRISPR-associated protein (Cas) system (CRISPRCas), providing valuable insights in the potential combinations of CRISPR-Cas system and nuclei acid nanotechnology. Prof. Zhu and Dr. Ma from Nanjing University reviewed DNA technology-assisted signal amplification strategies in electrochemiluminescence (ECL) bioanalysis and outlined the future trends of DNA nanotechnology in ECL realm.

As for the research articles, Prof. Luo's group at Qingdao University of Science and Technology developed a kind of visible light-responsive DNA thermotropic liquid crystals based on gold nanoparticles (Au NPs). The DNA and $\mathrm{Au}$ NPs were optimized to manipulate the photocurrent generation properties, which may facilitate the related investigations in biomedicine and biomaterials. Prof. Zuo and coworkers used DNA sequence mutation and native polyacrylamide gel electrophoresis to investigate the topology and conformations of biological G-quadruplexes. The proposed methodology with feasibility and low costs holds promise to uncover more unidentified G-quadruplex folds and functions. As an energy currency, adenosine triphosphate (ATP) plays pivotal roles in physiological environment. Harnessing the high affinity of ATP molecules towards the RNA aptamers, Prof. Xiang from Southwest University described a target recycling transcription of lighting-up 
aptamer strategy for detecting ATP in human serums in a label-free means with high sensitivity. Prof. Li from Zhengzhou University developed a fluorescent metal-organic frameworks (MOF)/aptamer nanoprobe to image and sense intracellular ATP by combining 2D Cu-MOF nanosheets and FAM-labelled ATP aptamer.

Despite the achievements being made, tackling the shortcomings of DNA technology still needs to be realized to promote its practical application. On one hand, the sensitivity and accuracy of DNA-based diagnostic platform should be further improved to adapt to complex environment both in vitro or in vivo. On the other hand, the long-term application of DNA materials in physiological environment ought to be guaranteed by improved stability that can protect DNA from biological degradation. Moreover, biosafety of DNAbased material regarding the immune clearance or immune rejection from organism should be carefully investigated to ensure the clinical translation.

This special issue only accounts for a small collection of DNA technology in the field of biosensing, bioimaging and biomedicine. We hope that it could be helpful for readers to get a knowledge of the current state of research in these fields. To conclude, we appreciate above-mentioned contributions in this special issue and would like to thank all the authors and reviewers for their efforts on this special issue.
In addition, we are grateful for the assistance from the Editorial Office of Journal of Analysis and Testing.

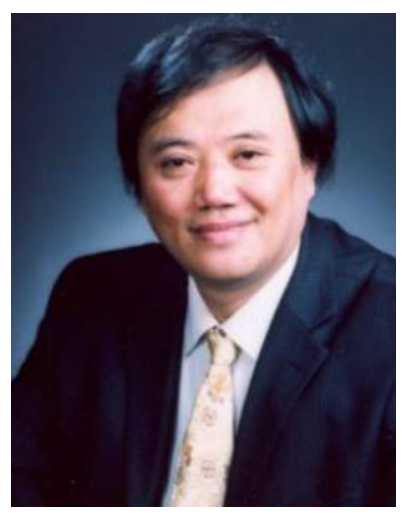

Jun-Jie Zhu is a full professor at Nanjing University. He got his BS degree in chemistry in 1984 and $\mathrm{PhD}$ degree in 1993 at Nanjing University respectively. $\mathrm{He}$ served as a postdoc fellow at Bar Ilan University, Israel (19981999). He became associate professor in 1996 and full professor in 2001 at Nanjing University. He has ever got National Distinguished Young Scholar in 2003 and was invited as FRSC in 2016. His research work focuses on Analytical Chemistry and Nano Science including nanobioanalytical chemistry, nanobioelectrochemistry, optical analysis of nanomaterials, bioapplication of nanomaterials, etc. He was ranked in the Elsevier's annual list of China's Most Cited Researchers in chemistry for 2014-2020 year. He is also 2018-2020 highly cited researchers from the Thomson Reuters highly cited researcher in Cross-Field category. He also serves as associate editors of "Analyst," "Current Smart Materials," and "Frontiers in Sensors." 